\title{
A SET OF AXIOMS FOR LINE GEOMETRY*
}

BY

\author{
E. R. HEDRICK AND LOUIS INGOLD
}

1. Introduction. This paper proposes a set of axioms for line geometry $\dagger$ based upon the line as an undefined element and intersection as an undefined relation between unordered pairs of elements. These same undefined concepts have been used in a set of axioms by Pieri.

The Pieri system, however, does not seem to accomplish all that could be desired in the way of simplicity, and there are several rather obvious redundancies in it which the authors of the present paper have been able to avoid.§

* Presented to the Society in a somewhat different form, December 1, 1911. At that time the paper of Pieri mentioned below was unknown to the authors.

$\dagger$ The precise types of geometry studied are discussed below.

$\ddagger$ Sui principi che regono la geometria delle rette, A t $\mathrm{t}$ i d ell a $\mathrm{Acc}$ a d e mi a d i Tor i n o, vol. 36 (1901), pp. 335-351. The relation used by Pieri is introduced first as a relation between ordered pairs of elements; but he states an axiom later to make the relation independent of the order.

$\S$ For reference, we give here the system of Pieri:

Postul $^{0}$ I. Il raggio e una classe non illusoria.

Postul $^{\ell}$ II. Ogni raggia incontra se stesso.

Postul $^{\ell}$ III. Essendo $a$ un raggio, esiste almeno un raggio, che taglia $a$ senza coincider $\operatorname{con} a$.

Postul ${ }^{\varrho}$ IV. Posto che $a, b$ siano raggi distinti, e che $a$ tagli $b$, si deduce che $b$ taglia $a$.

Df. Date le rette $a, b$ che s'incontrino senza confondersi, per fascio $a b$, s'intende la classe di tutti quei raggi, ognuno de quali s'incontra con ogni retta, che tagli al medesimo tempo $a$ e $b$. La stessa figura si rappresenta col simbolo ( $a b$ ).

Postul $^{\ell}$ V. Dal supposto, che due raggi $a$ e $b$ s'incontrino senza confondersi, e $c$ sia un raggio di ( $a b$ ) non coincidente con $a$, si deduce che $b$ appartiene ad ( $a c$ ).

Postul $^{\ell}$ VI. Qualunque volta due raggi $a$ e $b$ si tagliano senza confondersi, nel fascio ( $a b$ ) giace almeno un raggio diverso da $a$ e da $b$.

Postul $^{\varrho}$ VII. Dato che $a, b$ siano raggi distinti e l'un l'altro incidenti, dovra esistere un raggio che li tagli ambedue senza giacere in $(a b)$.

Df. Si chiama triraggio, o trilatispigolo, la figura costituita in tre rette, di cui ciascuna incontri ciascuna sepero non esiste alcun fascio di raggi che le contenga.

Postul $^{\varrho}$ VIII. Posto che i raggi $a, b$ e $c$ siano elementi d'un trilatispigolo, se $a^{\prime}$ e un ( $b c$ ) diverso da $b$ e da $c$, similmente $b^{\prime}$ un ( $c a$ ) diverso da $c$ e da $a$, deve esisteri almeno un raggio comune ai due fasci $\left(a a^{\prime}\right)$ e $\left(b b^{\prime}\right)$.

Postul $^{\varrho}$ IX. Se di due rette $a$ e $b$, che s'incontrano senza coincidere, ognuna e tagliata da due rette sghembe $c$ e $d$; allora ogni raggio che incontri al medisimo tempo $a$ e $b$, dovra tagliare uno almeno dei raggi $c, d$. 
In this paper a set of five axioms is introduced which are essentially equivalent to Pieri's system, and which seem to be quite as simple in statement and in application as those of Pieri. It is shown also that the resulting space is a general projective space, that is, the space is either properly or improperly projective according to the classification introduced by Veblen and Young.* This follows from the fact that the five axioms of this paper are equivalent to the two groups $A$ and $E$ of assumptions given by them.

The axioms are stated in terms of the undefined concepts line and intersection, and two defined secondary concepts, viz., a pencil of lines, and what we have called $a$ doublet of lines. These secondary concepts are defined in terms of line and intersection in what follows. After the axioms, a third secondary concept, a field of lines, is defined.

For the purpose of easy orientation, the reader may think of a field of lines either as a set of lines lying in a given projective plane, or as a set of lines passing through a given point of space. He may regard a pencil of lines as a set of lines of a plane through a given point of that plane. A doublet may be thought of as a set consisting of all the lines of a plane together with all the lines of space which pass through a given point of that plane. It should be noticed, however, that the concepts plane and point are not used in setting up the system; rather we have been able to define these concepts in terms of the fundamental notions line and intersection. The concepts field, pencil, and doublet seem particularly adapted to a treatment of line geometry and their early introduction makes possible a great simplification of the axioms.

The plan of the paper lends itself to extension to higher dimensions, although the generalization is not immediate. On some other occasion this extension will be treated.

2. The Axioms and their Independence. In what follows, the undefined elements (lines) will be denoted by small Roman letters. We shall presuppose nothing regarding the character of the undefined relation (intersection) except

Postul $^{\varrho} \mathrm{X}$. E ogni raggio, che incontri tutte e quattro le rette $a, b, c, d$ giace nel fascio $(a b)$.

Postul $^{\varrho}$ XI. Dati ancora due raggi $a$ e $b$ che s'incontrino senza coincidere, e date una retta $g$ che non tagli $a$ ne $b$, sara 'necessario che questa $g$ incontri un qualche raggio di $(a b)$.

Of these, Nos. I, III, V, VI, VII, VIII, IX, X, XI, are consequences of our axioms. No. II is unnecessary in the present system, since the relation of intersection is not defined to apply for a pair of identical lines. No. IV is not necessary in the system unless it is presumed that the relation of intersection depends on the order of the two elements as well as on the elements themselves.

It may be added that Pieri's V and VIII can be proved from his II, IV, VII, IX, X, and XI, and that the major content of his III and his VI can be proved from his XI.

* $A$ set of assumptions for projective geometry, American Journal of Mathem a t i c s, vol. 30 (1908), pp. 347-380; in particular, p. 352. These axioms are stated below in \$6. See also Veblen and Young, Projective Geometry, Ginn \& Co., 1910. 
that it holds or fails for any two distinct elements.* If the relation holds for two lines $a$ and $b$ we shall say that they intersect, or that $a$ meets $b$, or that $b$ meets $a$. If the relation fails, we shall say that $a$ and $b$ are skew, or that they are $a$ pair of skew lines.

Definition 1. If $a$ and $b$ are a pair of intersecting lines, the set of lines consisting of $a$ and $b$ together with all the lines that meet both $a$ and $b$ is called a doublet, and is denoted by the symbol [ $a b]$.

Definition 2. The set of lines of a doublet [ $a b]$ each of which meets every other line of the doublet $[a b]$ is called a pencil, and is denoted by the symbol $(a b)$.

In any doublet $[a, b]$ there is evidently just one pencil $(a, b)$.

Aхıом I. There exists at least one pair of intersecting lines.

Aхıмм II. In every doublet [ab] there is at least one line not belonging to the pencil $(a b)$.

Axıом III. Every line l of every doublet [ab] which is not in the pencil (ab) meets one and only one of any two skew lines of $[a b]$ both different from $l$.

Aхгом IV. Both of any two intersecting lines are skew to some third line. $\dagger$

Ахгом V. Every line meets at least one line of every pencil. $\ddagger$

We give the following examples to show the technical independence of these five axioms, in their order.

1. The elements are two lines; they do not meet. All the axioms except I are satisfied (vacuously).

2. The elements are the lines of one plane together with the five perpendiculars to that plane at the vertices of a certain regular pentagon of the plane. Intersection shall be interpreted as usual (projectively), except that the perpendiculars to the plane shall all be skew to each other. Axiom II is the only axiom violated.

3. The elements are the lines of an ordinary projective space and one extra line $l^{\prime}$. Intersection shall be interpreted as usual for all lines except $l^{\prime}$, while $l^{\prime}$ shall meet all lines that meet a given line $l$ of space and be skew to all other lines of space, including $l$ itself. Axiom III alone is violated.

4. The elements are the edges of a tetrahedron. Intersection is to be understood in the ordinary sense. All the axioms except IV are satisfied.

* It should be noticed that in this paper the undefined relation holds or fails for the set of objects $(a, b)$, not for the sequence $(a, b)$.

$\dagger$ It is necessary to assume this only for every pair of lines of which at least one belongs to a certain fixed pencil. It seems actually to defeat the desire for simplicity to remove this redundancy, but it may be done by stating IV as follows:

$I V^{\prime}$. If there exists a pair of intersecting lines, then there are two intersecting lines $a$ and $b$ such that, if either of any two intersecting lines $m$ and $n$ belongs to $(a b)$, a line $k$ skew to both $m$ and $n$ exists.

$\ddagger$ It is evident that this last axiom contains a redundancy, in that we already know that any line which meets either $a$ or $b$ meets at least one line of the pencil ( $a b)$. 
5. The elements are the lines of an ordinary projective space and one extra line $l^{\prime}$. Intersection shall have the usual meaning, except that $l^{\prime}$ shall be skew to every other line. All the axioms except $\mathrm{V}$ are satisfied.

Axiom I may be broken into two parts, as follows:

$\mathrm{I}_{1}$. There exists at least one line. (Pieri's I.)

$\mathrm{I}_{2}$. If there exists one line, there exists a pair of intersecting lines.*

These statements are independent of each other, and the system $I_{1}, I_{2}$, II, III, IV, V form a mutually independent system. For, the examples (2), (3), (4), (5), above, need not be altered; and (1) serves as an independence example for $I_{2}$. If there are no lines whatever, all but $I_{1}$ are satisfied.

Again, Axiom $\mathrm{I}_{2}$ may be divided into two parts, as follows:

$\mathrm{I}_{2}^{\prime}$. If there exists one line, there exists at least one other line.

$\mathrm{I}_{2}^{\prime \prime}$. If there exist tu'o lines, there exist two intersecting lines. $\dagger$

Axiom III can be readily broken into two parts by replacing in it the words one and only one by $\left(\mathrm{III}_{1}\right)$ the word one, or $\left(\mathrm{III}_{2}\right)$ the words not more than one.

3. Freliminary Definitions and Theorems. Before proceeding to any theorems, we shall define another secondary concept, a field of lines.

Definition 3. A set of lines of a doublet is called a field if (a) the set contains no pair of skew lines; $(b)$ every line of the doublet not in the set is skew to at least one line of the set.

Fields will sometimes be denoted by small Greek letters. As an illustration of the usefulness of this concept, we may state, by Axiom III, that the lines of a given doublet $[a b]$ not in a given field $\alpha$ of $[a b]$ are all skew to any line of $\alpha$ not in the pencil $(a b)$.

Theorem I. In any doublet there are tuo and only two fields.

By Axiom II, there exists at least one pair of skew lines $p$ and $q$ in any given doublet. All the lines of the doublet that meet $p$ meet each other, by Axiom III. Now $p$ is skew to all other lines of the doublet. Hence $p$ together with the lines of the doublet that meet $p$ constitute a field.

* It should be noticed that $\mathrm{I}_{2}$ is by no means equivalent to Pieri's III. But our $\mathrm{V}$ (or Pieri's XI) and $I_{2}$ suffice to prove his III.

$\dagger$ The possibility of dividing most axioms into component parts seems not to have been sufficiently emphasized. For example, the statement of $A_{1}$ of the Veblen-Young list ( $\$ 6$, below) is rather obviously composed of the two parts:

$A_{1}^{\prime}$. If $A$ and $B$ are distinct points, there is at least one line.

$A_{1}^{\prime \prime}$. If $A$ and $B$ are distinct points and if there is at least one line, there is a line containing both $A$ and $B$.

It is evident that $A_{1}^{\prime}$ is also a portion of $E_{1} . \quad \mathrm{A}_{1}^{\prime \prime}$ can be split into two parts in a similar manner, and so on ad infinitum. In fact it seems to be true that the existence of an infinite number of elements in any system at once renders it possible to arrange at least a countably infinite set of independent axioms whose content does not overlap, and which are all provable from any axioms that define that set. Hence it seems difficult to define conveniently the difference between single and multiple statements. 
Another field consists of $q$ and all the lines of the given doublet that meet $q$. There is no other field, since there is no line skew to both $p$ and $q$, by Axiom III.

Theorem 2. If a line $p$ of a doublet [ab] meets two skew lines $c$ and $k$ of that doublet, it belongs to the pencil $(a b)$.

For, since every pair of the set $a, b, p, c$ intersect, they belong to a field; likewise, $a, b, p, k$ belong to a field. These fields are different, since $c$ and $k$ cannot belong to the same field. Hence $p$ lies in both fields and therefore belongs to the pencil $(a b)$.

Corollary. The pencil $(a b)$ contains the lines $a$ and $b$.

Theorem 3. If $p$ and $q$ are distinct lines of the pencil $(a b)$, the doublet $[p q]$ is identical with the doublet $[a b]$.

For, $p$ and $q$ belong to both fields of the doublet [ab ] ; hence a line meeting $a$ and $b$ meets $p$ and $q$; and therefore the doublet $[a b]$ is contained in the doublet $[p q]$.

Again, $a$ and $b$ belong to $[p q]$ and each meets at least one pair of skew lines that belongs to $[a b]$ and therefore also to $[p q]$; hence, by Theorem 2 , $a$ and $b$ belong to the pencil $(p q)$.

Interchanging $a$ and $b$ with $p$ and $q$ in the argument above, it is seen that any line of $[p q]$ is also a line of $[a b]$.

Corollary 2. If a line meets two lines of a pencil, it meets every line of the pencil.

Corollary 3. If $c$ is a line of the pencil $(a b)$ and $c \neq b$, then $a$ is a line of the pencil $(b c)$.

Theorem 4. If $c$ is a line of the doublet $[a b]$ which does not belong to the pencil $(a b)$, that field of the doublet $[a b]$ which contains consists of $a, b, c$ and all the lines that meet $a, b$, and $c$.

Let $p$ and $q$ be any two lines that meet $a, b$, and $c$; suppose that $p$ and $q$ are skew. Then, by Theorem 2, since $c$ meets $p$ and $q, c$ belongs to the pencil $(a b)$, contrary to hypothesis. Hence $p$ and $q$ intersect and therefore belong to the field of the doublet $[a b]$ which contains $c$.

Definition 4. If $c$ is a line of the doublet [ab] which does not belong to the pencil $(a b)$, the three lines $a, b, c$ form a triad.

Theorem 5. There is one and only one field containing the lines of a given triad.

This follows immediately from Theorem 4 and Definition 2.

It will be convenient to denote the field determined by the lines $a, b, c$ of a triad by $(a b c)$.

Theorem 6. If two distinct pencils belong to the same field, they have one and only one line in common.

Let the distinct pencils $(a b)$ and $(c d)$ belong to a field $\alpha$; then there is a line $l$ meeting $a$ and $b$ but not belonging to $\alpha$. The lines $c$ and $d$ cannot both 
belong to the pencil ( $a b)$; for, by Theorem 3, $(a b)$ would then coincide with $(c d)$. Hence $l$ does not meet all lines of $(c d)$. By Axiom V, $l$ meets at least one line $m$ of $(c d)$. Hence by Corollary $2, l$ meets one and only one line $m$ of $(c d)$. The line $m$ belongs to ( $a b)$, since $l$ meets only those lines of $\alpha$ which are lines of $(a b)$. No other line can be common to the two pencils, since $l$ meets every line of $(a b)$ and only the line $m$ of $(c d)$.

Theorem 7. The lines of a field that meet a line not belonging to the field form a pencil.

Let $p$ be a line not belonging to the field $\alpha$ of the doublet [ab]. If $p$ meets $a$ and $b$, then $p$ meets every line of the pencil $(a b)$, and the theorem is proved.

If $p$ does not meet both $a$ and $b$, there is at least one line $c$ of the pencil ( $a b$ ) which $p$ meets, by Axiom V; also there is a line $l$ of the field $\alpha$ which does not belong to the pencil ( $a b)$. Then $\alpha$ is identical with the field ( $a b l$ ) by Theorem 5. Suppose $p$ does not meet $a$. The line $p$ meets one line $d$ of the pencil ( $a l$ ). Hence $p$ meets two lines $c$ and $d$ of the field $\alpha$, so that $p$ meets all lines of the pencil $(c d)$.

There is no other line of the field $\alpha$ which meets $p$. For if $p$ meets all lines of the pencil ( $c d$ ) and one other line of the field $\alpha$, then $p$ belongs to the field $\alpha$.

4. Classification of Fields. We shall now show that fields fall into two main classes, for which a principle of duality holds. Later we shall identify these two classes of fields with points and planes of ordinary geometry.*

Definition 5. Two distinct fields are said to be incident to one another if they have in common all the lines of a pencil.

Theorem 8. If two distinct fields are incident to the same field they have in common one and only one line.

If $\alpha$ and $\beta$ are both incident to $\phi$, there is a pencil $P$ common to $\alpha$ and $\phi$ and a pencil $Q$ common to $\beta$ and $\phi$.

The pencils $P$ and $Q$ have one and only one line $l$ in common. Let $p$ and $q$ be two other lines belonging to $P$ and $Q$, respectively. Then $l, p$, and $q$ are three lines of $\phi$ forming a triad; also $p$ belongs to $\alpha$ and $q$ belongs to $\beta$. Let $k$ be any line common to $\alpha$ and $\beta$. Then $k$ meets $l, p$, and $q$. Hence $k$ belongs to $\phi$, and therefore to each of the pencils $P$ and $Q$. It follows that $k$ is identical with $l$.

Definition 6. Two fields which have one and only one line in common are said to be similar to each other; otherwise they are not similar, except that a field is said to bo similar to itself.

Theorem 9. If a field $\alpha$ is not similar to a field $\psi$ and if $\psi$ is incident to a field $\phi$, then $\alpha$ is similar to $\phi$.

* Such a classification, whose purpose is to realize the well-known duality of point and plane in line geometry, will obviously be a part of any treatment of the subject. Thus Pieri, loc. cit., gives a treatment similar to that of this paper, but his proofs are of course essentially different. 
1. If $\alpha$ is incident to $\psi$, there is one and only one line common to $\alpha$ and $\phi$, by Theorem 8 ; hence $\alpha$ is similar to $\phi$.

2. If $\alpha$ is not incident to $\psi$, let $l$ be any line of $\alpha$ which does not belong to $\phi$ (if there is no such line, $\alpha$ and $\phi$ are identical and therefore similar). Denote by $P$ the pencil common to $\phi$ and $\psi$. Then $l$ meets one and only one line $m$ of $P$; let $r$ be another line of $P$. The lines of $\alpha$ which meet $m$ form a pencil $A$; $r$ meets at least one line $s$ of $A ; s$ meets $m$ and $r$; hence $s$ belongs to the field $\phi$. For; the line $s$ cannot belong to $\psi$, since it belongs to $\alpha$. The lines $l, m$, and $s$ form a triad; $\alpha$ is incident to the field (lms). The field $\phi$ also is incident to (lms). Hence $\alpha$ and $\phi$ have only the line $s$ in common, so that $\alpha$ is similar to $\phi$, as was to be proved.

TheOREM 10. If each of two fields $\alpha$ and $\beta$ is similar to a field $\phi, \alpha$ is similar to $\beta$.

Let $a$ be common to $\alpha$ and $\phi$, and $b$ common to $\beta$ and $\phi$. If $a$ coincides with $b$, there is no other line common to $\alpha$ and $\beta$. For, suppose $k$ is such a line, so that $\alpha$ and $\beta$ are incident. Then, since $k$ does not belong to $\phi, k$ meets $\phi$ in a pencil $A$. Let $a^{\prime}$ be a line of this pencil different from $a$. Then $a, a^{\prime}$, and $k$ form a triad. The field $\left(a a^{\prime} k\right)$ contains the pencil $(a k)$ which is common to $\alpha$ and $\beta$. Hence ( $a a^{\prime} k$ ) is identical with either $\alpha$ or $\beta$, and therefore $\alpha$ or $\beta$ has the pencil $\left(a a^{\prime}\right)=A$ in common with $\phi$, contrary to hypothesis.

If $a$ is different from $b$, then at least $a$ meets $b$, since they belong to the same field $\phi$. Let $\psi$ be the field incident to $\phi$ containing $a$ and $b$. Then $a$ meets $\beta$ in a pencil $B$. Let $b^{\prime}$ denote a line of $B$ different from $b$. The line $b^{\prime}$ meets $a$ and $b$ and does not belong to $\phi$, since $b^{\prime}$ belongs to $\beta$. Hence $b^{\prime}$ belongs to $\psi$ and therefore $\beta$ has the pencil $\left(b b^{\prime}\right)$ in common with $\psi$. Similarly, $\alpha$ has a pencil in common with $\psi$. Hence, by Theorem $8, \alpha$ is similar to $\beta$.

Theorem 11. If each of two fields $\alpha$ and $\beta$ is not similar to a field $\phi, \alpha$ is similar to $\beta$.

Let $\psi$ be a field incident to $\phi$. Then, by Theorem 9, $\alpha$ and $\beta$ are both similar to $\psi$. Hence by Theorem $10, \alpha$ is similar to $\beta$.

THEOREM 12. If a field $\alpha$ is similar to a field $\phi$ and if another field $\beta$ is not similar to $\phi$, then $\alpha$ is not similar to $\beta$.

For, if $\alpha$ were similar to $\beta$, then, by Theorem 10, we would have $\beta$ similar to $\phi$, contrary to hypothesis.

By Theorems 10,11, 12, we can separate all fields into two classes, those in one class being all that are similar to a given field $\phi$, and those of the other class being the fields which are not similar to $\phi$. Any two belonging to the same class are similar to each other, any two belonging to different classes are not similar to each other. 
5. Identification of Fields with Points and Planes. We shall call the fields of one of the classes just mentioned planes, and those of the other class points. Since these two classes of fields possess a complete duality, a distinction as to which fields are to be called points and which are to be called planes is impossible.

If a point and a plane are incident to one another, we say that the point lies in the plane or that the plane passes through the point, or contains the point.

If a line belongs to a plane, we say that the line lies in the plane or that the plane passes through the line.

We say that two intersecting lines $a$ and $b$ meet in $a$ point and also that they determine a plane.

Other expressions commonly used in geometry, such as "the line joining two points," "the line of intersection of two planes," etc., may also be defined in an obvious way.

It is now possible to prove many theorems of projective geometry relating to the intersections of lines and planes and the collinearity of points; for example, Desargues's theorem regarding two perspective triangles could be proved; and, again, the quadrangle construction for the harmonic conjugate of a given point with respect to two others on the same line is clearly possible.

6. Comparison with Other Systems of Axioms. In conclusion we compare the axioms of this paper with a complete system of axioms for projective geometry, so that it may be seen what further axioms are needed to complete the usual theory: The system chosen for this purpose is that of Veblen and Young cited above. The following groups $\mathrm{A}$ and $\mathrm{E}$ of their assumptions will be proved to be equivalent to our Axioms I-V.

$\mathrm{A}_{1}$. If $A$ and $B$ are distinct points, there is at least one line containing both $A$ and $B$.

A2. If $A$ and $B$ are distinct points, there is not more than one line containing both $A$ and $B$.

$\mathrm{A}_{3}$. If $A, B, C$ are points not belonging to the same line, and if a line $l$ contains a point $D$ of a line joining $B$ and $C$ and a point $E$, distinct from $D$, of a line joining $C$ and $A$, then the line $l$ contains a point $F$ of a line joining $A$ and $B$.

$\mathrm{E}_{0}$. There are at least three points on every line.

$\mathrm{E}_{1}$. There exists at least one line.

$\mathbf{E}_{2}$. It is not true that every point lies on every line.

$\mathrm{E}_{3}$. It is not true that every point lies on every plane.*

* Under at least one interpretation of these statements, $E_{1}$ is a logical consequence of $E_{2}$, and both $E_{1}$ and $E_{2}$ are consequences of $E_{3}$. The somewhat altered statements in Veblen and Young, Projective Geometry, p. 24, appear to be independent, however. 
$\mathrm{E}_{3}{ }^{\prime}$. If $S$ is a three-space, every point lies in $S$.

In this system the plane and the three-space are defined in terms of the undefined elements point and line as follows:

Definition. If $P, Q, R$ are three points not on the same line, and $l$ is a line joining $Q$ and $R$, the class $S_{2}$ of all points such that every point of $S_{2}$ is collinear with $P$ and some point of $l$ is called the plane determined by $P$ and $l$. If $P, Q, R, T$ are four points not in the same line or plane, and if $\alpha$ is a plane containing $Q, R$, and $T$, the class $S_{3}$ of all points such that every point of $S_{3}$ is collinear with $P$ and some point of $\alpha$ is called the three-space determined by $P$ and $\alpha$.

Assumptions $A_{1}$ and $A_{2}$ follow from our axioms by the definition of similar fields.

To prove $A_{3}$, let $a$ be common to the (point) fields $C$ and $B$, and let $b$ and $c$ be the lines common to the (point) fields $C$ and $A$, and $A$ and $B$, respectively. Then $a, b$, and $c$ form a triad (triangle). Let $\phi$ be the (plane) field determined by $a, b$, and $c$. If a line $l$ meets $a$ and $b$, and if the (point) field $D$ determined by $a$ and $l$ and the (point) field $E$ determined by $b$ and $l$ are distinct, then $a, b$, and $l$ do not all three belong to a (point) field. Hence $l$ belongs to the (plane) field ( $a b c)$, so that $l$ meets $c$, and the (point) field determined by $l$ and $c$ is a point of a line $c$ joining $A$ and $B$.

$\mathrm{E}_{0}$ follows from I, II, IV, and $\mathrm{V}$ in an obvious manner.

$\mathrm{E}_{1}$ follows from $\mathrm{I}$; or indeed from $\mathrm{E}_{2}$, as remarked above.

$\mathrm{E}_{2}$ follows from $\mathrm{E}_{3}$; or it may be proved directly as follows. Consider a line $l$ on which there is a point $P$, and let $a$ and $b$ be two lines passing through $P$. We shall denote the field which defines the point $P$ by $\alpha$. The lines $a$ and $b$ determine also a (plane) field $\beta$. Let $c$ be a line of this plane not belonging to the pencil $(a b)$. Then $c$ meets both $a$ and $b$; the three points determined by $a$ and $b, b$ and $c, c$ and $a$, respectively, do not all three lie on the same line. Hence $\mathrm{E}_{2}$ is proved.

To prove $\mathrm{E}_{3}$, consider the plane $\alpha$ of any plane triad $a, b, c$. There is a line $p$, not lying in $\alpha$, which passes through the point $P$ determined by $a$ and $b$. The lines $p$ and $a$ determine a plane which contains a line $q$ not passing through the point $P$. The lines $p$ and $q$ determine the point $R$. Now $R$ is distinct from $P$, for otherwise $q$ would pass through $P$; hence $R$ does not lie in $\alpha$, for then $p$ would also lie in $\alpha$.

To prove $\mathrm{E}_{3}$, let $S_{3}$ be a three-space determined by a point $P$ and a plane $\alpha$ containing the intersecting lines $a$ and $b$; and let $Q$ denote a point. Let $l$ be a line common to $P$ and $Q$. By V, $l$ meets at least one line $c$ of the pencil $(a b)$. Hence $Q$ is collinear with $P$ and the point determined by $l$ and $c$ of the plane $\alpha$. Therefore $Q$ is a point of $S_{3}$ by definition.

It can also be shown that I, II, III, IV, and V follow from the assumptions $A$ and $\mathrm{E}$. 
7. Conclusion. In order to complete the entire theory, it is only necessary to follow precisely the steps taken by Veblen and Young, for example, beyond their assumptions $A$ and $E$, in the paper referred to above, and those taken by Veblen in a later paper.* The remaining assumptions of the VeblenYoung system are evidently independent of our set. Hence there seems to be no essential reason for pursuing the details of this discussion further.

Columbia, Mo.,

January, 1914.

* On the definition of the multiplication of irrational numbers, A m e r i c a $\mathbf{n}$ o u r $\mathrm{n}$ a 1 of M a t h e m a t i c s, vol. 34 (1912), pp. 211-214. 